\title{
The metabolic syndrome of fructose-fed rats: Effects of long-chain polyunsaturated $\omega 3$ and $\omega 6$ fatty acids. V. Post-mortem findings
}

\author{
ZOHEIR MELLOUK ${ }^{1}$, KARIM LOUCHAMI ${ }^{2}$, EMELINE HUPKENS $^{2}$, \\ ABDULLAH SENER $^{2}$, DALILA AIT YAHIA ${ }^{1}$ and WILLY J. MALAISSE ${ }^{2}$ \\ ${ }^{1}$ Department of Biology, Es-Sénia University, Es-Sénia 31000, Oran, Algeria; \\ ${ }^{2}$ Laboratory of Experimental Hormonology, Université Libre de Bruxelles, B-1070 Brussels, Belgium
}

Received June 1, 2012; Accepted September 17, 2012

DOI: $10.3892 / \mathrm{mmr} .2012 .1091$

\begin{abstract}
The present study deals with the possible effects of dietary $\omega 3$ and $\omega 6$ fatty acids upon the metabolic syndrome found in rats exposed for 8 weeks to a diet containing $64 \%$ (w/w) D-fructose instead of starch. Fructose-fed rats were found to display a modest increase in plasma albumin and protein concentration and more pronounced increases in plasma urea, creatinine, phospholipids, triglycerides and cholesterol concentrations, glycated hemoglobin concentration and liver contents of cholesterol, triglycerides and phospholipids. The plasma concentrations of HDL-cholesterol, calcium and iron were decreased, however, in the fructose-fed rats. In general, the partial substitution of sunflower oil by either safflower oil or salmon oil opposed the metabolic perturbations otherwise associated with the fructose-induced metabolic syndrome in the fructose-fed rats, with salmon oil demonstrating particular efficacy. Consideration is given to the possible biological determinants of these perturbations and their attenuation in rats exposed to safflower or salmon oil.
\end{abstract}

\section{Introduction}

The first four articles in this series concerned intraperitoneal glucose tolerance tests (1), the time course of changes in food intake, body weight, plasma glucose and insulin concentrations and insulin resistance (2), the secretory behaviour of isolated pancreatic islets (3) and the metabolism of D-glucose in isolated pancreatic islets (4) in fructose-fed rats. Comparisons were made between the results for control female rats exposed from the 8th week after birth and for the ensuing 8 weeks to

Correspondence to: Professor Willy J. Malaisse, Laboratory of Experimental Hormonology, Université Libre de Bruxelles, 808 Route de Lennik, Brussels B-1070, Belgium

E-mail: malaisse@ulb.ac.be

Key words: metabolic syndrome, fructose-fed rats, long-chain polyunsaturated $\omega 3$ and $\omega 6$ fatty acids a diet containing $64 \%(\mathrm{w} / \mathrm{w})$ starch and 5\% (w/w) sunflower oil (Ssun rats) and female rats exposed over the same period to a diet containing $64 \% \mathrm{D}$-fructose and $5 \%$ sunflower oil (Fsun rats) or 3.4\% sunflower oil mixed with $1.6 \%$ salmon oil (Fsal rats) or safflower oil (Fsaf rats). The selection of these experimental conditions was based on an extensive examination of the relevant literature, as documented in a review study dealing mainly with the influence of nutritional factors on the fructose-induced metabolic syndrome (5). The present report extends these observations to a first set of post-mortem investigations dealing inter alia with: the percentage of glycated hemoglobin in blood and liver glucokinase activity relating to carbohydrate metabolism; plasma concentration and liver content of cholesterol, triglycerides and phospholipids in respect to lipid metabolism; and protein, albumin, urea and creatinine concentrations in plasma in respect to nitrogen-rich compounds.

\section{Materials and methods}

The four groups of female Wistar rats used in the present study were the same as those defined in the first report in this series. At sacrifice, blood was sampled by cardiac puncture following overnight fasting.

The methods used for the measurement of the plasma concentration of albumin (2), blood concentration of glycated hemoglobin (2), liver protein content (6) and hepatic glucokinase activity (7) have been described previously. The kits used for the measurement of the plasma concentration of urea and creatinine (Biocon, Vöhl-Marienhagen, Germany), calcium (Spinreact S.A., Girona, Spain), iron (Randox Laboratories Ltd., Crumlin, UK), plasma and liver triglycerides and phospholipids (Spinreact S.A.) plasma and liver cholesterol (Biolabo, Maizy, France), plasma HDL-cholesterol (Biocon) were obtained from the sources cited in parentheses.

The plasma albumin concentration (2) and percentage of glycated hemoglobin (2) are listed in the present report for purpose of comparison. All results are presented as the mean value \pm SEM (standard error of the mean) together with the number of individual determinations (n). The statistical significance of a difference between mean values was assessed using the Student's t-test. 
Table I. Plasma data.

\begin{tabular}{|c|c|c|c|c|}
\hline & Ssun $(n=6)$ & Fsun $(n=6)$ & Fsal $(\mathrm{n}=5)$ & Fsaf $(n=6)$ \\
\hline Albumin (g/l) & $39.2 \pm 0.23$ & $42.00 \pm 0.24$ & $40.26 \pm 0.24$ & $41.67 \pm 0.15$ \\
\hline Urea $(\mathrm{mM})$ & $2.29 \pm 0.22$ & $3.04 \pm 0.09$ & $2.74 \pm 0.09$ & $2.84 \pm 0.09$ \\
\hline Creatinine (mg/dl) & $1.25 \pm 0.13$ & $1.88 \pm 0.25$ & $1.52 \pm 0.18$ & $1.75 \pm 0.22$ \\
\hline Calcium (mM) & $2.21 \pm 0.03$ & $1.75 \pm 0.01$ & $2.36 \pm 0.02$ & $2.38 \pm 0.02$ \\
\hline Iron $(\mu \mathrm{M})$ & $12.33 \pm 1.33$ & $8.86 \pm 0.85$ & $15.28 \pm 1.22$ & $10.15 \pm 1.13$ \\
\hline Phospholipids (mM) & $1.20 \pm 0.04$ & $2.50 \pm 0.06$ & $1.56 \pm 0.05$ & $1.69 \pm 0.05$ \\
\hline Triglycerides (mM) & $3.19 \pm 0.05$ & $5.12 \pm 0.18$ & $3.55 \pm 0.15$ & $4.86 \pm 0.17$ \\
\hline Total cholesterol (mM) & $3.22 \pm 0.04$ & $4.15 \pm 0.03$ & $3.35 \pm 0.24$ & $3.87 \pm 0.14$ \\
\hline HDL-cholesterol (mM) & $2.82 \pm 0.11$ & $2.15 \pm 0.19$ & $3.05 \pm 0.36$ & $2.33 \pm 0.08$ \\
\hline
\end{tabular}

Table II. Blood data.

\begin{tabular}{lcccc}
\hline & Ssun $(\mathrm{n}=6)$ & Fsun $(\mathrm{n}=6)$ & Fsal $(\mathrm{n}=5)$ & Fsaf $(\mathrm{n}=6)$ \\
\hline Hemoglobin $(\mathrm{mg} / \mathrm{ml})$ & $474 \pm 74$ & $532 \pm 82$ & $483 \pm 68$ & $522 \pm 77$ \\
Glycated hemoglobin $(\%)$ & $5.81 \pm 0.02$ & $13.67 \pm 0.13$ & $8.76 \pm 0.02$ & $12.11 \pm 0.02$ \\
\hline
\end{tabular}

Table III. Liver data.

\begin{tabular}{|c|c|c|c|c|}
\hline & Ssun & Fsun & Fsal & Fsaf \\
\hline Protein $(\mu \mathrm{g} / \mathrm{mg}$ wet wt. $)$ & $209 \pm 8^{a}$ & $204 \pm 17^{\mathrm{a}}$ & $192 \pm 18^{b}$ & $210 \pm 14^{\mathrm{a}}$ \\
\hline Glucokinase activity (nmol/mg wet wt. per $30 \mathrm{~min}$ ) & $6.05 \pm 0.27^{\mathrm{b}}$ & $6.37 \pm 0.21^{\mathrm{a}}$ & $8.77 \pm 0.86^{\mathrm{b}}$ & $6.78 \pm 0.24^{\mathrm{a}}$ \\
\hline Cholesterol ( $\mu \mathrm{mol} / \mathrm{g}$ wet wt.) & $32.50 \pm 0.66^{\mathrm{a}}$ & $44.41 \pm 0.36^{\mathrm{a}}$ & $35.89 \pm 0.37^{\mathrm{b}}$ & $38.72 \pm 0.22^{\mathrm{a}}$ \\
\hline Triglycerides ( $\mu \mathrm{mol} / \mathrm{g}$ wet wt.) & $23.39 \pm 0.34^{\mathrm{a}}$ & $36.85 \pm 0.33^{\mathrm{a}}$ & $28.56 \pm 0.48^{b}$ & $34.44 \pm 0.51^{\mathrm{a}}$ \\
\hline Phospholipids ( $\mu \mathrm{mol} / \mathrm{g}$ wet wt.) & $18.62 \pm 0.48^{\mathrm{a}}$ & $32.48 \pm 0.25^{\mathrm{a}}$ & $22.72 \pm 0.33^{\mathrm{b}}$ & $28.35 \pm 0.31^{\mathrm{a}}$ \\
\hline
\end{tabular}

${ }^{\mathrm{a}} \mathrm{n}=6 ;{ }^{\mathrm{b}} \mathrm{n}=5$.

\section{Results}

Plasma data. The mean plasma concentrations of albumin, urea, creatinine, phospholipids, triglycerides and total cholesterol were all higher $(\mathrm{P}<0.07$ or less $)$ in the Fsun rats than in the Ssun rats (Table I), such a difference being, in relative terms, most pronounced in the case of urea, creatinine, phospholipids, triglycerides and total cholesterol. The plasma concentrations of calcium, iron and HDL-cholesterol, however, were lower in the Fsun rats than in the Ssun rats $(\mathrm{P}<0.06$ or less).

The six variables listed in Table I which yielded higher mean values in the Fsun rats than in the Ssun rats displayed a comparable pattern when the results recorded in the fructose-fed rats were compared with those of the starch-fed animals. Thus, in the Fsun, Fsal and Fsaf rats, the measurements averaged, respectively, $148.0 \pm 6.3(\mathrm{n}=36 ; \mathrm{P}<0.001)$, $114.9 \pm 3.3(n=30 ; P<0.001)$ and $130.6 \pm 4.0 \%(n=36 ; P<0.001)$ of those in the Ssun rats $(100.0 \pm 1.9 \% ; n=36)$. In this respect, the mean results recorded in the Fsal and Fsaf rats were significantly lower $(\mathrm{P}<0.025$ or less) than those in the Fsun rats. The values in the Fsal rats were also significantly lower $(\mathrm{P}<0.005)$ than those in the Fsaf rats.

Relative to the differences between the Fsun and Ssun rats for these six variables $(100.0 \pm 6.7 \% ; n=36)$, those found between the Fsal or Fsaf rats and Ssun rats averaged 33.6 \pm 7.0 $(\mathrm{n}=30 ; \mathrm{P}<0.001)$ and $71.8 \pm 7.0 \%(\mathrm{n}=36 ; \mathrm{P}<0.005)$, respectively. That is, the increments recorded in the Fsaf rats averaged, for the same six variables, $272.7 \pm 34.2 \%(\mathrm{n}=36 ; \mathrm{P}<0.001)$ of the mean corresponding increments in the Fsal rats $(100.0 \pm 30.9 \%$; $\mathrm{n}=30$ ).

A comparable situation prevailed for the three variables, plasma HDL-cholesterol, calcium and iron concentrations, which had a lower value in the Fsun rats than in the Ssun rats. Indeed, relative to the mean value recorded in the latter rats $(100.0 \pm 3.6 \% ; n=18)$, those determined in the Fsun, Fsal and Fsaf rats averaged 74.8 $\pm 3.1(\mathrm{n}=18 ; \mathrm{P}<0.001), 113.0 \pm 5.4$ $(\mathrm{n}=15 ; \mathrm{P}<0.05)$ and $90.9 \pm 4.2 \%(\mathrm{n}=18 ; \mathrm{P}>0.11)$, respectively. The mean results recorded in the Fsal and Fsaf rats were thus significantly higher $(\mathrm{P}<0.005$ or less $)$ than those in the Fsun rats. Moreover, the values collected in the Fsal rats were also 
A

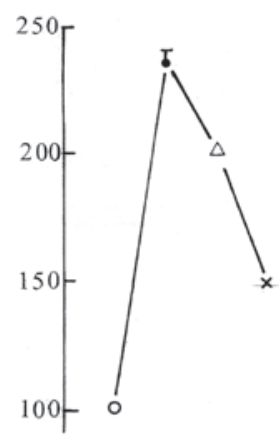

B
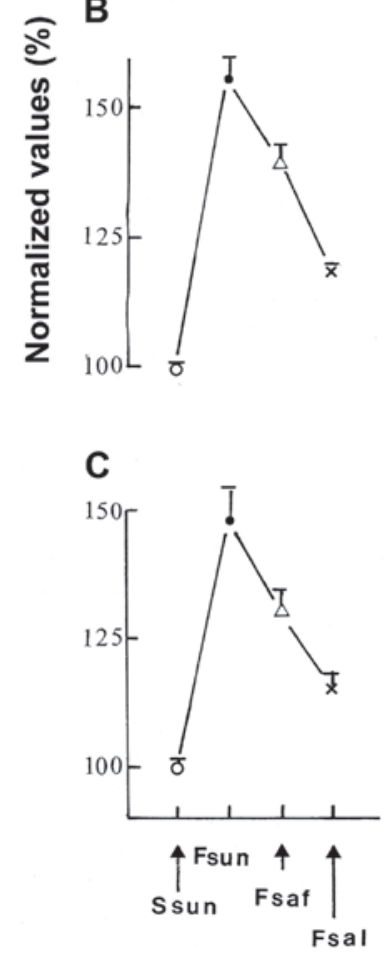

D

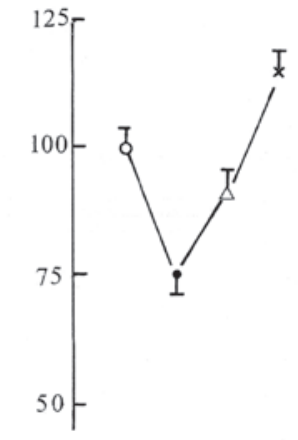

E

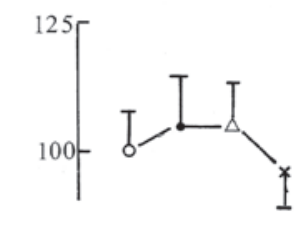

$\mathbf{F}$

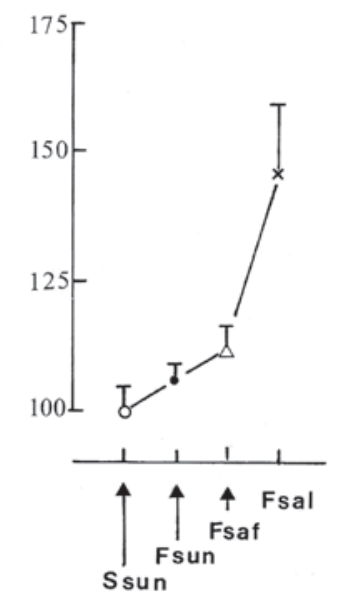

Figure 1. Metabolic parameters in Fsun $(\bullet)$, Fsaf $(\triangle)$ and Fsal (x) rats. Mean values ( \pm SEM) for (A) the percentage of glycated hemoglobin, (B) liver cholesterol, triglycerides and phospholipid content, (C) plasma concentration of albumin, urea, creatinine, phospholipids, triglycerides and total cholesterol, (D) plasma concentration of calcium, iron and HDL-cholesterol, (E) blood hemoglobin concentration and liver protein content and (F) liver glucokinase activity in Fsun, Fsaf and Fsal rats are expressed relative to the mean corresponding values found in Ssun rats $(\mathrm{O})$. They refer to (A and F) 5-6 individual data, (E) 10-12 individual data, (B and D) 15-18 individual data and (C) 30-36 individual data.

significantly higher $(\mathrm{P}<0.004)$ than those in the Fsaf rats. In the latter rats, they were not significantly different $(\mathrm{P}>0.11)$ from those in the Ssun rats, whilst those in the Fsal rats were higher $(\mathrm{P}<0.05$ or less $)$ than those in the Ssun rats.

Blood data. The concentration of hemoglobin in the blood did not differ significantly among the four groups of rats, with an overall mean value of $503 \pm 36 \mathrm{mg} / \mathrm{ml}(\mathrm{n}=23)$. The percentage of glycated hemoglobin, however, was significantly higher $(\mathrm{P}<0.001)$ in the fructose-fed than in the starch-fed rats (Table II). In the former rats, it again displayed a Fsun $>$ Fsaf $>$ Fsal hierarchy $(\mathrm{P}<0.001)$.
Liver data. The protein content of liver homogenates, expressed relative to wet weight, did not differ significantly among the four groups of rats (Table III), with an overall mean value of $204 \pm 7 \mu \mathrm{g} / \mathrm{mg}$ wet wt. $(\mathrm{n}=23)$. The data concerning the protein content of the liver were confirmed in an independent assay in which no significant difference ( $\mathrm{P}>0.20$ or more) was found among the mean values recorded in the four groups of rats. In the latter assay, the protein content of the heart or kidney also failed to differ among the four groups of rats, the data recorded in Fsun, Fsal and Fsal rats averaging, respectively, $107.3 \pm 5.8(n=12), 106.7 \pm 5.4(n=10)$ and $100.9 \pm 9.1 \%(n=10)$ of the corresponding mean values in the Ssun rats $(100.0 \pm 8.6 \%$; $\mathrm{n}=10$ ). Within the same assay, no significant differences were detected among the four groups of rats in terms of the protein content of the parametrial or visceral adipose tissue. The absolute values determined in adipose tissue were, however, one order of magnitude lower than in the liver, representing in the parametrial and visceral adipose tissue no more than $9.4 \pm 1.1$ and $11.9 \pm 1.2 \%$ of the mean value in liver samples (100.0 $\pm 6.5 \% ; n=23$ in all three cases).

As judged from triplicate measurements of liver glucokinase activity conducted in each of two separate assays, with a correlation coefficient between the latter two assays of +0.922 $(n=22 ; P<0.001)$, the results recorded in the Fsun rats did not differ significantly ( $\mathrm{P}>0.2$ or more) from those found in the Ssun or Fsaf rats (Table III). However, the values found in the Fsaf rats appeared higher $(\mathrm{P}<0.06)$ than those recorded in the Ssun rats. Finally, in the Fsal rats, the activity of glucokinase exceeded that found in the Ssun $(\mathrm{P}<0.02)$, Fsun $(\mathrm{P}<0.02)$ and Fsaf rats $(\mathrm{P}<0.05)$.

The mean values for the liver content of cholesterol, triglycerides and phospholipids in the four groups of rats are listed in Table III. These three variables displayed a comparable hierarchy in the four groups of rats, the overall mean values in the Fsun, Fsaf and Fsal rats averaging, respectively, 156.2 \pm 3.8 $(n=18), 139.5 \pm 3.6(n=18)$ and $118.2 \pm 1.8 \%(n=15)$ of the mean corresponding values in the Ssun rats $(100.0 \pm 1.1 \% ; n=18)$. The latter four mean values differed significantly ( $\mathrm{P}<0.004$ or less) from one another.

\section{Discussion}

The results recorded in the present study are shown in Fig. 1. It documents that, for the majority of variables, specifically the percentage of glycated hemoglobin (Fig. 1A), liver cholesterol, triglyceride and phospholipid content (Fig. 1B) and plasma concentration of albumin, urea, creatinine, phospholipids, triglycerides and total cholesterol (Fig. 1C), the measurements recorded in the Fsun rats were invariably higher than those determined in the Ssun rats. In the fructose-fed rats, the substitution of part of the sunflower oil by an equal amount of either safflower oil or salmon oil minimized the relative magnitude of such increases, with salmon oil having the greatest minimizing effect. Conversely, the substitution of starch by D-fructose in the diet containing sunflower oil decreased the plasma concentration of calcium, iron and HDL-cholesterol (Fig. 1D). The effect of D-fructose was again opposed by the substitution of some of the sunflower oil by either safflower oil or salmon oil, the latter having the stronger effect. Two variables, that is, the blood hemoglobin concentration and liver protein content, 

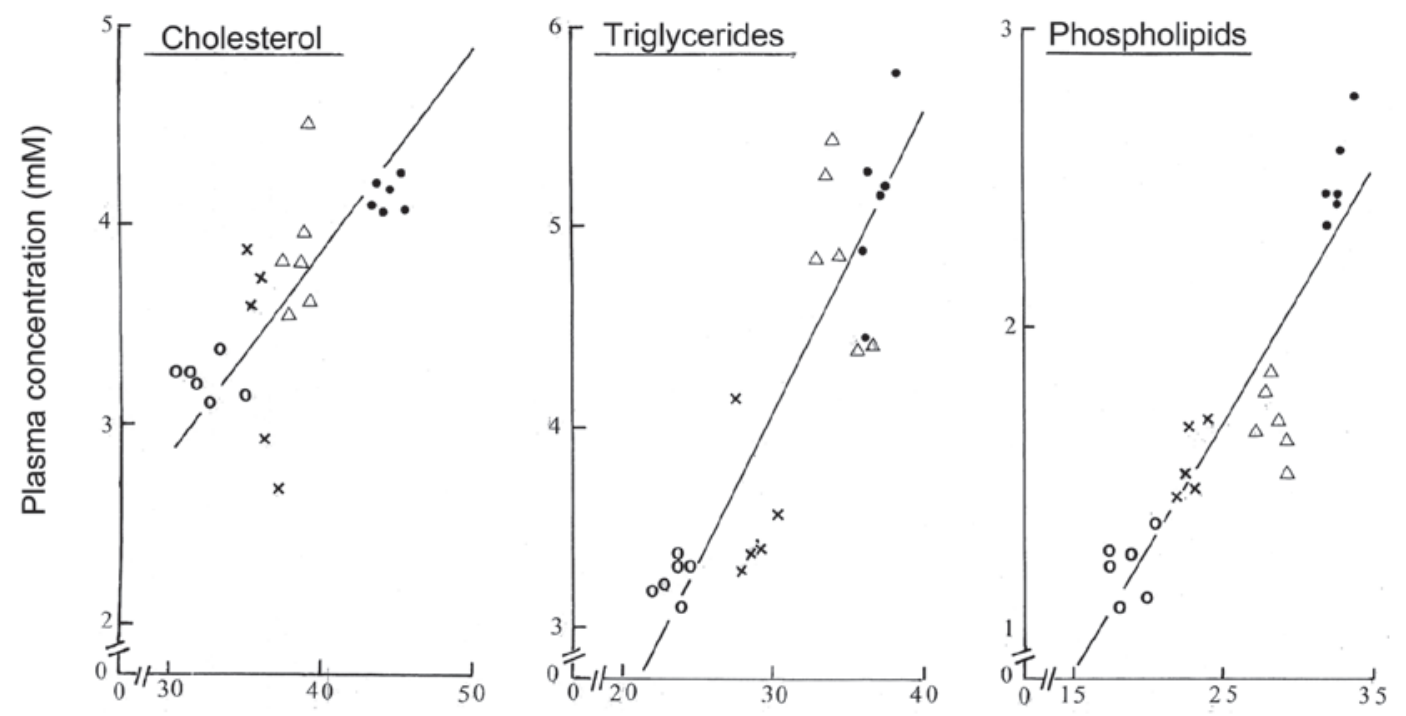

Liver content ( $\mu \mathrm{mol} / \mathrm{g}$ wet wt.)

Figure 2. Comparison between the plasma concentration and liver content of cholesterol, triglycerides and phospholipids in Ssun ( $\mathrm{O})$, Fsun (•), Fsal (x) and Fsaf $(\Delta)$ rats. The oblique lines correspond to the regression lines with slopes equal to the square root of the quotient $\left(\Sigma y^{2} / \Sigma x^{2}\right)$ between the sum of the squared individual differences from mean values in plasma concentration $\left(\Sigma \mathrm{y}^{2}\right)$ and the sum of the squared individual differences from mean values in liver content $\left(\Sigma \mathrm{x}^{2}\right)$.

were not significantly affected by the dietary manipulations (Fig. 1E). Finally, the activity of liver glucokinase was markedly higher in the Fsal rats than in the other three groups of rats (Fig. 1F). Thus, in several respects, the partial substitution of sunflower oil by either safflower oil or salmon oil corrected, at least to some extent, the biochemical perturbations found in the metabolic syndrome of the fructose-fed rats (Fig. 1A-C) and, on occasion, even increased the parameter under consideration above the level found in the control Ssun rats (Fig. 1D and F).

The determinants responsible for these diet-induced changes in metabolic variables remain, to some extent, a matter of speculation, as recently reviewed elsewhere (5). The following considerations should not be ignored, however. In terms of carbohydrate metabolism and as judged from the percentage of glycated hemoglobin, the present findings are consistent with the well-known perturbation of glucose homeostasis in fructose-fed rats, currently attributed to an increase in hepatic glucose output and peripheral insulin resistance (5). This supports the view that the dietary supply of $\omega 3$ fatty acids to the Fsal rats and, to a lesser extent, that of $\omega 6$ fatty acids, in this case mainly C18:2 $\omega 6$ to the Fsal rats (1) may oppose such an unfavorable effect of the fructose-rich diet (5). Incidentally, in the Fsal rats, the glycogen content of the liver did not differ $(98.5 \pm 7.5 \% ; n=6)$ from that in the Ssun rats $(100.0 \pm 8.3 \%$; $\mathrm{n}=6)$, whilst being decreased $(\mathrm{P}<0.007)$ to $71.8 \pm 4.9 \%(\mathrm{n}=11)$ of the same reference value in the other fructose-fed rats (data not shown). The major novel information provided by the present study in respect to carbohydrate metabolism relates to the measurements of liver glucokinase activity. The results of such measurements, as given in Table III, document a higher activity of glucokinase in the liver of the Fsal rats than in the other 3 groups of rats $(\mathrm{P}<0.05$ or less $)$. The higher liver glucokinase activity in the Fsal rats, as compared with either the Fsun or Fsaf rats, is reminiscent of the higher glucokinase activity found in the pancreatic islet homogenates of control rats, as compared with second generation rats depleted in long- chain polyunsaturated $\omega 3$ fatty acids, a difference tentatively ascribed to inhibition of the enzyme by endogenous long-chain fatty acyl-coenzyme A in the $\omega 3$-depleted rats (7). Indeed, in the present study, the Fsal rats were the sole fructose-fed animals exposed to a diet containing sizeable amounts of long-chain polyunsaturated $\omega 3$ fatty acids (1), this coinciding with a lower liver triglyceride content $(\mathrm{P}<0.001)$ in the Fsal rats than in the Fsun or Fsaf rats. Furthermore, in the fructosefed rats, a significant negative correlation prevailed between the individual values for liver glucokinase activity and liver triglyceride content $(\mathrm{r}=-0.580 ; \mathrm{n}=17 ; \mathrm{P}<0.02)$.

In terms of lipid metabolism, the salient findings comprised: the increased plasma concentration and liver content of cholesterol, triglycerides and phospholipids, but decreased plasma concentration of HDL-cholesterol in the Fsun rats, as compared with the Ssun rats; and the partial or complete correction of these perturbations in the Fsaf and Fsal rats, particularly in the latter. The internal consistency of these findings is further documented by the negative correlation found at the individual level between the total cholesterol concentration and that of HDL-cholesterol in plasma $(\mathrm{r}=-0.537$; $\mathrm{n}=23$; $\mathrm{P}<0.01)$. Likewise, the likely relationship between liver and plasma lipids is supported by the finding of close correlations between the individual values for the liver content of cholesterol, triglycerides and phospholipids and the plasma concentration of the same lipids (Fig. 2). The correlation coefficient in those three cases averaged $0.846 \pm 0.055(n=3)$ with, in all cases, a probability well below 0.001 ( $n=23$ in all three cases). Notably, the individual negative xy products represented no more than $1.6 \pm 0.8 \%(n=3)$ of the corresponding positive xy products, no negative individual xy product being observed between the liver content and plasma concentration of triglycerides.

No clear difference in the protein content of the liver, heart, kidney or adipose tissue was observed among the four groups of rats. Nevertheless, the plasma albumin concentration was somewhat higher $(\mathrm{P}<0.02$ or less $)$ in each group of fructose-fed 
rats than in the control starch-fed rats (Table I). It was significantly lower, however, in the Fsal rats than in the Fsun or Fsaf rats $(\mathrm{P}<0.001)$. Likewise, the plasma protein concentration was slightly higher $(\mathrm{P}<0.05)$ in the fructose-fed rats than in the control starch-fed rats (data not shown). The correlation between the individual values for plasma albumin and protein concentrations was close to achieving statistical significance $(\mathrm{r}=+0.389 ; \mathrm{n}=23 ; \mathrm{P}<0.07)$.

The urea and creatinine plasma concentrations were increased in the Fsun rats, with a trend towards normalization in the Fsaf and Fsal rats. Relative to the corresponding mean values found in Ssun rats $(100.0 \pm 5.5 \% ; n=12)$, the measurements for the Fsun rats averaged $141.6 \pm 10.1 \%(\mathrm{n}=12 ; \mathrm{P}<0.002)$ and decreased to $132.0 \pm 8.9 \%(n=12)$ in the Fsaf rats and $120.6 \pm 7.0 \%(n=10)$ in the Fsal rats. The latter two mean values were significantly higher $(\mathrm{P}<0.04$ or less), however, than that determined in the Ssun rats. These findings may indicate incipient nephropathy in the fructose-fed rats.

Finally, the plasma concentration of calcium and iron also underwent dual changes. Relative to the mean value in Ssun rats $(100.0 \pm 5.2 \% ; n=12)$, that in the Fsun rats was decreased $(\mathrm{P}<0.001)$ to $75.5 \pm 3.5 \%(\mathrm{n}=12)$ and was distinctly different $(\mathrm{P}<0.01$ or less $)$ from those in Fsaf $(95.1 \pm 5.8 \%, \mathrm{n}=12)$ and Fsal rats $(115.5 \pm 5.5 \% ; n=10)$. The latter value was not significantly higher $(\mathrm{P}<0.06)$ than that recorded in the control Ssun rats. Changes in the plasma contribution of albumin are unlikely to act as determinants of changes in the plasma concentration of calcium, the correlation coefficient between these two variables $(\mathrm{r}=-0.390 ; \mathrm{n}=23 ; \mathrm{P}<0.07)$ being negative. As an alternative hypothesis, it may be speculated that the decrease in plasma calcium and iron concentration indicates an impaired intestinal absorption of these metals. To our knowledge, however, an enteropathy in animal models of diabetes mellitus has only been well-documented in type 1 diabetic animals such as BB rats $(8-10)$, in which case it was proposed that the enteropathy plays a role in the development of type 1 diabetes, rather than resulting from it (11).

In summary, the present set of post-mortem data collected in rats exposed for 8 weeks to a fructose-rich diet and distinct oils confirms certain classical findings and reveals some unexpected features of the metabolic syndrome prevailing in fructose-fed rats, whilst duly documenting the favorable effect of diets enriched with selected long-chain polyunsaturated $\omega 3$ and $\omega 6$ fatty acids on minimizing the fructose-induced perturbations in several metabolic variables.

\section{Acknowledgements}

We are grateful to C. Demesmaeker for secretarial help.

\section{References}

1. Mellouk Z, Hachimi Idrissi T, Louchami K, Hupkens E, Malaisse WJ, Ait Yahia D and Sener A: The metabolic syndrome of fructose-fed rats: effects of long-chain polyunsaturated $\omega 3$ and $\omega 6$ fatty acids. I. Intraperitoneal glucose tolerance test. Int J Mol Med 28: 1087-1092, 2011.

2. Mellouk Z, Hachimi Idrissi T, Louchami K, Hupkens E, Sener A, Ait Yahia D and Malaisse WJ: The metabolic syndrome of fructose-fed rats: effects of long-chain polyunsaturated $\omega 3$ and $\omega 6$ fatty acids. II. Time course of changes in food intake, body weight, plasma glucose and insulin concentrations and insulin resistance. Int J Mol Med 29: 113-118, 2012.

3. Mellouk Z, Zhang Y, Bulur N, Louchami K, Malaisse WJ, Ait Yahia D and Sener A: The metabolic syndrome of fructose-fed rats: effects of long-chain polyunsaturated $\omega 3$ and $\omega 6$ fatty acids. III. Secretory behaviour of isolated pancreatic islets. Int J Mol Med 29: 285-290, 2012.

4. Mellouk Z, Zhang Y, Bulur N, Louchami K, Sener A, Ait Yahia D and Malaisse WJ: The metabolic syndrome of fructose-fed rats: effects of long-chain polyunsaturated $\omega 3$ and $\omega 6$ fatty acids. IV. D-glucose metabolism in isolated pancreatic islets. Int J Mol Med 29: 291-293, 2012.

5. Boukortt FO, Madani Z, Mellouk Z, Louchami K, Sener A and Ait Yahia D: Nutritional factors and fructose-induced metabolic syndrome. Metab Funct Res Diab 4: 18-34, 2011.

6. Lowry $\mathrm{OH}$ and Passonneau JV (eds): A Flexible System of Enzymatic Analysis. Academic Press, New York, p174, 1972.

7. Zhang Y, Bulur N, Peltier S, Carpentier YA, Malaisse WJ and Sener A: Long-chain fatty acyl-coenzyme A-induced inhibition of glucokinase in islets from rats depleted in long-chain polyunsaturated $\omega 3$ fatty acids. Cell Biochem Funct 26: 233-237, 2008.

8. Courtois P, Sener A, Scott FW and Malaisse WJ: Disaccharidase activity in the intestinal tract of Wistar-Furth, BBc and BBdp rats. Br J Nutr 91: 201-209, 2004.

9. Courtois P, Sener A, Scott FW and Malaisse WJ: Peroxidase activity in the intestinal tract of Wistar-Furth, BBc and BBdp rats. Diabetes Metab Res Rev 20: 305-314, 2004.

10. Graham S, Courtois P, Malaisse WJ, Rozing J, Scott FW and Mowat AM: Enteropathy precedes type 1 diabetes in the BB rat. Gut 53: 1437-1444, 2004.

11. Malaisse WJ, Courtois P and Scott FW: Insulin-dependent diabetes and gut dysfunction: the BB rat model. Horm Metab Res 36: 585-594, 2004. 